\title{
Statistical Diagnosis for Random Right Censored Data Based on Kaplan-Meier Product Limit Estimate
}

\author{
Shuling Wang1, Xiaohong Deng1, Lin Zheng² \\ ${ }^{1}$ Department of Fundamental Course, Air Force Logistics College, Xuzhou, China \\ ${ }^{2}$ School of Statistics and Applied Mathematics, Anhui University of Finance and Economics, Bengbu, China \\ Email: 155328313@qq.com
}

Received 20 April 2014; revised 15 May 2014; accepted 2 June 2014

Copyright (C) 2014 by authors and Scientific Research Publishing Inc. This work is licensed under the Creative Commons Attribution International License (CC BY). http://creativecommons.org/licenses/by/4.0/

(c) (i) Open Access

\begin{abstract}
In this work, we consider statistical diagnostic for random right censored data based on K-M product limit estimator. Under the definition of K-M product limit estimator, we obtain that the relation formula between estimators. Similar to complete data, we define likelihood displacement and likelihood ratio statistic. Through a real data application, we show that our proposed procedure is validity.
\end{abstract}

\section{Keywords}

Random Right Censorship, Kaplan-Meier Product-Limit Estimator, Empirical Likelihood, Outliers, Influence Analysis

\section{Introduction}

Statistical diagnosis developed in the mid-1970s, which is a new statistical branch.

In the course of development of the past 40 years, most scholars have studied the data into a convenient and effective statistical model. For example, the diagnosis and influence analysis of linear regression model has been fully developed (R. D. Cook and S. Weisberg [1], Bocheng Wei, Guobin Lu \& Jianqing Shi [2]); The varing coefficient model is a useful extension of classical linear model. Regarding the varying coefficient model, especially for the B-spline estimation of parameter, diagnosis and influence analysis have some results (Cai, Z., Fan, J., Li, R. [3], Fan, J., Zhang, W. [4]). However, all the above results are obtained under the uncensored case. In many applications, some of the responses and/or covariants may not be observed, but are censored. For censored data, the usual statistical techniques for complete data situations are not readily applicable. Because there are too many hypothesis, it is easy to lose information. 
As we all known, the distribution function of a random variable $\mathrm{X}$ contains all of the probabilistic information about X. Hence this paper tries to use non-parametric maximum likelihood estimate (NPMLE) [5] of distribution function in follow-up study.

The rest of the paper is organized as follows. The right censoring and K-M product limit estimator is introduced in Section 2. Outlier diagnosis and influence analysis are presented in Section 3. An example is given to illustrate our results in Section 4.

\section{Right Censoring and Kaplan-Meier Product Limit Estimator}

Here, the distribution of a real-valued random variables $X_{i}$ is of direct interest. For each $X_{i}$ there is a $Y_{i} \in R$. This $Y_{i}$ may be random. If $X_{i} \leq Y_{i}$ we observe $X_{i}$, otherwise $X_{i}$ is censored to $\left(Y_{i}, \infty\right)$. We say that $X_{i}$ is right censored by $Y_{i}$. Let $Z_{i}=\min \left\{X_{i}, Y_{i}\right\}$ and $\delta_{i}=1_{X_{i} \leq Y_{i}}$. For example, $X_{i}$ could be survival time after an operation, with $Y_{i}$ the time from the operation to the end of the study.

The idea of the K-M product limit estimator is given by the conditional probability. Let $t_{i} \leq t_{i+1}$ :

$$
\begin{aligned}
F\left(t_{i}\right) & =1-S\left(t_{i}\right)=1-P\left(T>t_{i}\right)=1-P\left(T>t_{i}, T>t_{i-1}\right)=1-P\left(T>t_{i} \mid T>t_{i-1}\right) P\left(T>t_{i-1}\right) \\
& =1-P\left(T>t_{i} \mid T>t_{i-1}\right) P\left(T>t_{i-1} \mid T>t_{i-2}\right) \cdots P\left(T>t_{0}=0\right)
\end{aligned}
$$

We assume that at the start of the study all subjects were alive, so $P\left(T>T_{0}=0\right)=1$. The conditional probability is

$$
P\left(T>t_{i}, T>t_{i-1}\right)=\frac{r_{i}-d_{i}}{r_{i}} P\left(T>t_{i}, T>t_{i-1}\right)=\frac{r_{i}-d_{i}}{r_{i}},
$$

where $r_{i}$ is the number of subjects at risk in the study at the time $t_{i}$, and $d_{i}$ is the number of subject dying at time $t_{i}$. The Kaplan-Meier estimator of CDF is

$$
\hat{F}=1-\prod_{i \mid t_{i} \leq t} \frac{r_{i}-d_{i}}{r_{i}} .
$$

\section{Statistical Diagnostic}

For complete data, diagnostic measures of outlier contain case deletion and mean shift, influence statistics contain Cook's distance, W-K statistic, covariance ratio statistic, AP statistic, likelihood distance and so on. Similarly, we derive several diagnostic measures for right censored data.

\subsection{Outlier Diagnosis}

Let $\hat{F}(i)$ is the K-M product limit estimator of distribution function after case deletion, then there are lemma

$$
\hat{F}(i) \hat{F}-\hat{F}(i)=\left\{\begin{array}{cc}
\frac{d_{i}}{r_{i}} \prod_{\substack{j t_{j} \leq t \\
(j \neq i)}} \frac{r_{j}-d_{j}}{r_{j}}, & t_{i} \leq t \\
0, & t_{i}>t
\end{array} .\right.
$$

Proof: By the definition of K-M product limit estimator, there are

$$
\hat{F}=1-\prod_{j \mid t_{j} \leq t} \frac{r_{j}-d_{j}}{r_{j}}
$$

and

$$
\hat{F}_{(i)}=1-\prod_{\substack{j \mid t_{j} \leq t \\(j \neq i)}} \frac{r_{j}-d_{j}}{r_{j}}
$$

Since, when $t_{i} \leq t$, there are 


$$
\begin{aligned}
\hat{F}-\hat{F}(i) & =\left(1-\prod_{j \mid t_{j} \leq t} \frac{r_{j}-d_{j}}{r_{j}}\right)-\left(1-\prod_{\substack{j \mid t_{j} \leq t \\
(j \neq i)}} \frac{r_{j}-d_{j}}{r_{j}}\right) \\
& =\prod_{\substack{j \mid t_{j} \leq t \\
(j \neq i)}} \frac{r_{j}-d_{j}}{r_{j}}-\prod_{j \mid t_{j} \leq t} \frac{r_{j}-d_{j}}{r_{j}} \\
& =\left(1-\frac{r_{i}-d_{i}}{r_{i}} \prod_{\substack{j \mid t_{j} \leq t \\
(j \neq i)}} \frac{r_{j}-d_{j}}{r_{j}}=\frac{d_{i}}{r_{i}} \prod_{\substack{\left.j \mid t_{j} \leq t \\
j \neq i\right)}} \frac{r_{j}-d_{j}}{r_{j}} .\right.
\end{aligned}
$$

when $t_{i}>t, \hat{F}-\hat{F}_{(i)}=0$ is obviously.

From the lemma, we can construct the relation formula between estimators, which is the foundation of discussion.

\subsection{Influence Analysis}

\subsubsection{Likelihood Displacement}

The likelihood function is defined as

$$
L(F)=\prod_{i=1}^{n} F\left(\left\{Z_{i}\right\}\right)^{\delta_{i}} F\left(\left(Z_{i}, \infty\right)\right)^{1-\delta_{i}}
$$

where $\delta_{i}=1_{X_{i} \leq Y_{i}}, Z_{i}=\min \left(X_{i}, Y_{i}\right)$, which can be computed from the $Z_{i}$ and $\delta_{i}$ without knowing the $Y_{i}$ from uncensored $X_{i}$.

Likelihood displacement is the method for measuring influence, which is advanced by Cook and Weisberg in 1982, which is advanced from the view of data fitting. Considering the influence of deleting the $i$-th case. Then, the likelihood displacement can be expressed as follows

$$
L D_{i}(F)=2\left[L(F)-L\left(F_{(i)}\right)\right] .
$$

\subsubsection{Likelihood Ratio Statistic}

For complete data, there is likelihood ratio statistic. Similarly, we define the likelihood ratio statistic for censored data based on K-M product limit estimator as follows

$$
R_{i}=\frac{L\left(F_{(i)}\right)}{L(F)}=\frac{\prod_{j=1, j \neq i}^{n} F_{(i)}\left(\left\{Z_{j}\right\}\right)^{\delta_{j}} F_{(i)}\left(\left(Z_{j}, \infty\right)\right)^{1-\delta_{j}}}{\prod_{i=1}^{n} F\left(\left\{Z_{i}\right\}\right)^{\delta_{i}} F\left(\left(Z_{i}, \infty\right)\right)^{1-\delta_{i}}}
$$

\section{Numerical Studies}

(Vicious Tumour Data) In this section, we consider an example as the illustration for the above results. Considering a clinical research trial data (see Andersen [6]). There are 205 cancer patients who have been treated in Odense university hospital and tracked until the end of 1977. The survival time of some individuals due to death or end of the trial for other reasons were censored. Wang Qihua [7] ultized a linear semi-parametric model to fit these test data. Wang Shuling et al. [8] ultized a nonparametric regression model with random right censorship to fit the data of 126 female patients. Now we consider the first twenty data, calculate the likelihood function by MATLAB and obtain $L(F)=0.0000003$. The originality data and the other results are in following Table 1.

Where $L\left(F_{(i)}\right)$ is likelihood function after deleting the $i$-th case, the results of $L D_{i}$ and $R_{i}$ are as follows. 
Table 1 . The originality data and the value of $L\left(F_{(i)}\right)$.

\begin{tabular}{|c|c|c|}
\hline$Z_{i}$ & $\delta_{i}$ & $L\left(F_{(i)}\right)$ \\
\hline 0.0099 & 0 & 0.0000003 \\
\hline 0.0232 & 0 & 0.0000003 \\
\hline 0.0279 & 1 & 0.00000102 \\
\hline 0.0295 & 1 & 0.00000102 \\
\hline 0.0355 & 0 & 0.00000043 \\
\hline 0.0386 & 1 & 0.00000086 \\
\hline 0.0469 & 1 & 0.00000086 \\
\hline 0.0667 & 1 & 0.00000086 \\
\hline 0.0817 & 1 & 0.00000086 \\
\hline 0.0826 & 0 & 0.00000077 \\
\hline 0.0833 & 1 & 0.00000074 \\
\hline 0.0858 & 1 & 0.00000074 \\
\hline 0.0869 & 1 & 0.00000074 \\
\hline 0.0872 & 1 & 0.00000074 \\
\hline 0.0982 & 1 & 0.00000074 \\
\hline 0.1055 & 1 & 0.00000074 \\
\hline 0.1156 & 1 & 0.00000074 \\
\hline 0.1252 & 1 & 0.00000074 \\
\hline 0.1271 & 1 & 0.00000074 \\
\hline 0.1312 & 1 & 0.00000074 \\
\hline
\end{tabular}

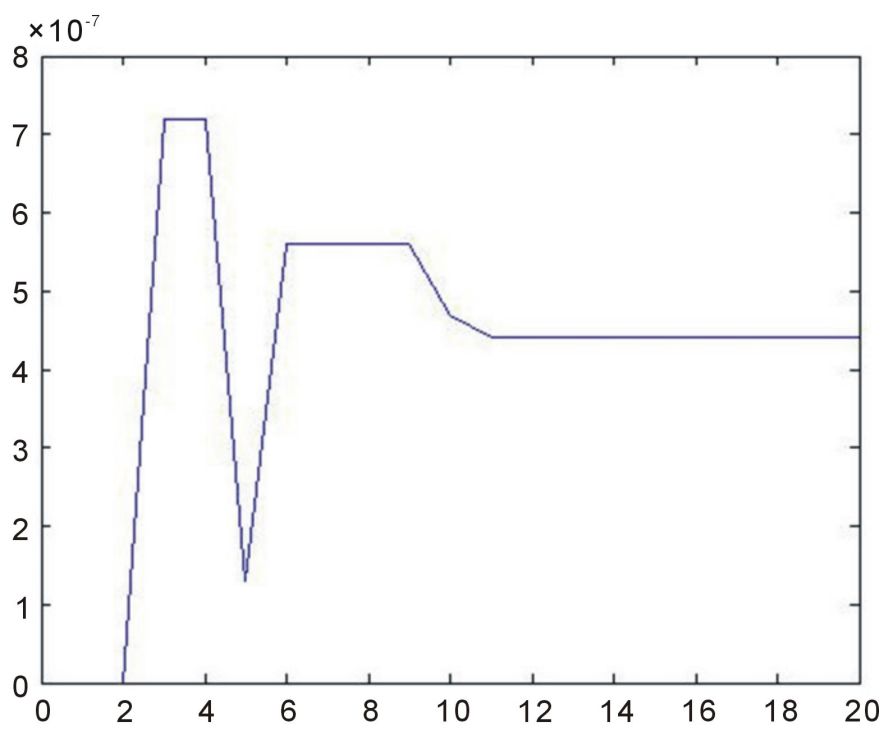

Figure 1. The value of LDi. 


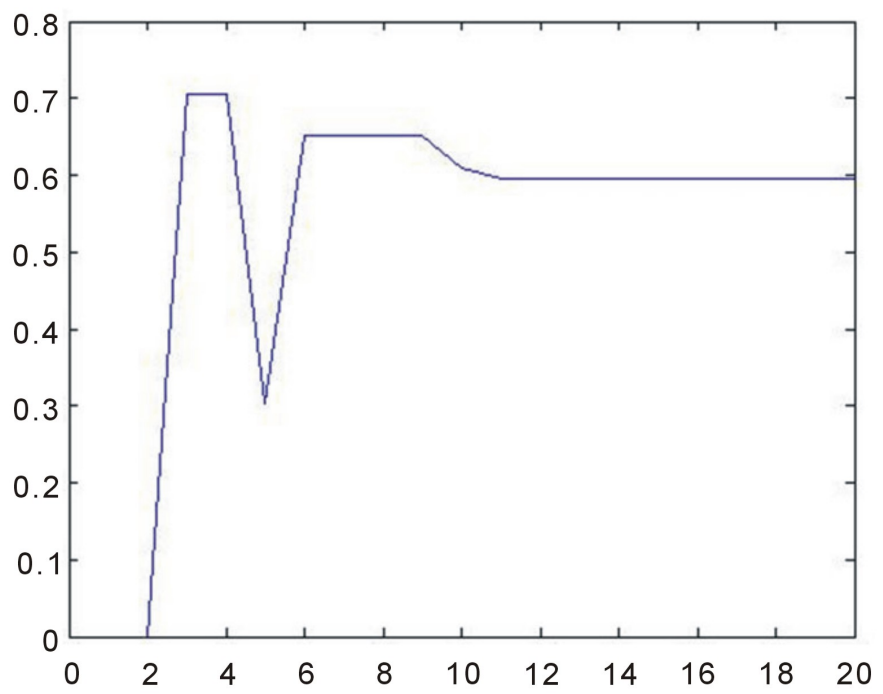

Figure 2. The value of Ri.

Figure 1 and Figure 2 show that the first, second, third, fourth and fifth data are outliers. Indeed, this result is similar to Wang Shuling et al. [8].

\section{References}

[1] Cook, R.D. and Weisberg, S. (1982) Residuals and Influence in Regression. Chapman and Hall, New York.

[2] Wei, B.C., Lu, G.B. and Shi, J.Q. (1990) Statistical Diagnostics. Publishing House of Southeast University, Nanjing.

[3] Cai, Z., Fan, J. and Li, R. (2000) Efficient Estimation and Inferences for Varying-Coefficient Models. Journal of American Statistical Association, 95, 888-902. http://dx.doi.org/10.1080/01621459.2000.10474280

[4] Fan, J. and Zhang, W. (2008) Statistical Methods with Varying Coefficient Models. Statistics and Its Interface, 1, 179-195. http://dx.doi.org/10.4310/SII.2008.v1.n1.a15

[5] Owen, A. (2001) Empirical Likelihood. Chapman and Hall, New York. http://dx.doi.org/10.1201/9781420036152

[6] Andersen, P.K., Borgan, O., Gill, R.D. and Keiding, N. (1993) Statistical Models Based on Counting Processes. Springer-Verlag, New York.

[7] Wang, Q.H. (2006) Analysis of Survival Data. Science Press, Beijing.

[8] Wang, S.L., Feng, Y. and Liu, X.B. (2010) Statistical Diagnostics of Nonparametric Regression Model with Random Right Censorship. Journal of Hefei University of Technology (Natural Science), 33, 470-473. 
Scientific Research Publishing (SCIRP) is one of the largest Open Access journal publishers. It is currently publishing more than 200 open access, online, peer-reviewed journals covering a wide range of academic disciplines. SCIRP serves the worldwide academic communities and contributes to the progress and application of science with its publication.

Other selected journals from SCIRP are listed as below. Submit your manuscript to us via either submit@scirp.org or Online Submission Portal.
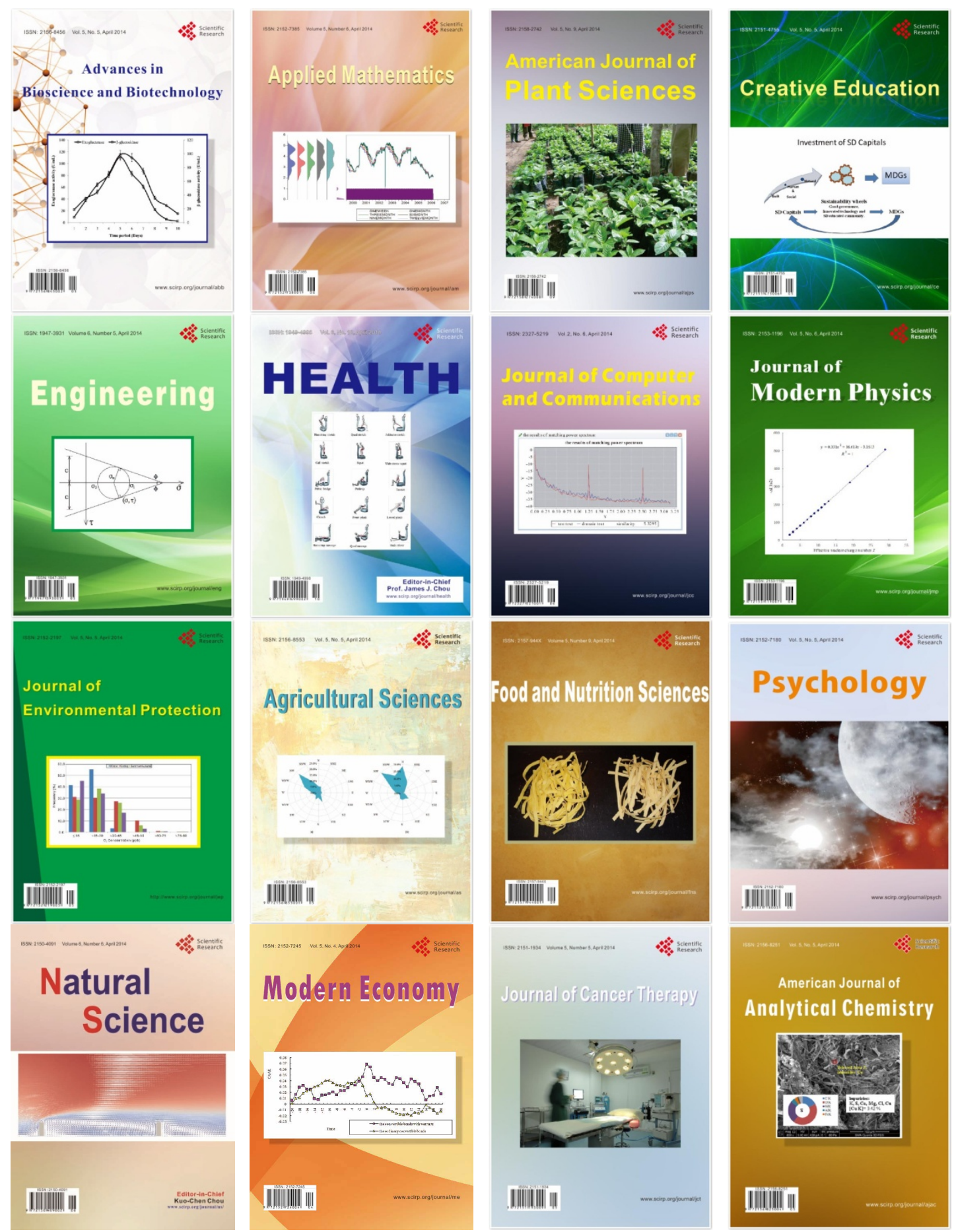